\title{
New Investigation Perspectives in Psychology and Behav- ioral Sciences: Informational Activity and Psychological/ Behavioral Expression of the Cognitive Centers of Con- sciousness
}

\section{Editorial}

Volume 2 Issue 4- 2021

\author{
Author Details \\ Florin Gaiseanu* \\ Science and Technology of Information, Bucharest (Romania) and Barcelona (Spain) \\ *Corresponding author \\ Florin Gaiseanu, Science and Technology of Information, Bucharest (Romania) and Barcelona (Spain)
}

Article History

Received: December 14, 2021 Accepted: December 22, 2021 Published: December 30, 2021

\section{Editorial}

Psychology investigates basically how do we think, and the behavioral science how do we manifest the thinking. Both of them search the correlation between the neural activity, the mechanisms of thinking and how the results of thinking are expressed. The psychology and behavior sciences are mainly based on the following theories and corresponding concepts [1]: (i) the cognitive theory, focused on the perception, memory and retrieval of remembrances; (ii) the humanistic theory, centered on individual and his/her distinct feelings, actions and self-image; (iii) the behavior theory, based on the stimulus-response during the interaction with the environmental factors; (iv) the psychodynamic theory, based on Freud's concepts [2], which claims the importance of the subconscious mind on body "desires" like hunger, sexuality and the self-image role in interaction with the others; (v) the biological theory regards the biological substrate of behavior, centered mainly to the nervous dysfunctions and mental health. None of these theories however do encompasses by itself the full psyche/behavior spectrum of human features on the entire range of complexity. The recent approach of consciousness and cognition from the perspective of the informational concepts succeeds to cover this lack.

Indeed, as it has been shown recently, information is an essential player of the human organism and of all other living structures $[3,4]$, concurring together with matter and energy to their structuration $[5,6]$. The living structures, particularly human, are connected to matter and information [7], as it is schematically shown in Figure 1. The human psychological traits are based on seven directives, coming from the life requirements itself: the need of connection to the environmental information is assured by the center of acquisition and storing of information (CASI), the interaction and communication with the environment by the reactive/decisional support of the center of decision and command (CDC), the reactive sentience and immediate action, as a feeling form of connection with internal/external reality (acceptance/rejection Good/Bad YES/NO Bit-type reaction) by means of the info-emotional system (IES), the management of the metabolic processes by the maintenance informational system (MIS), the biological reproduction by genetic transmission system (GTS), the inherited genetic information by the info-genetic generator (IGG), and the info-"navigation" among the multitude of informational data of internal/external sources by info-connection/selection (IC) pole (Figure 1 left side).

Human receives information from external and internal sources through sensors, each of them converting the physical information (light, sound, chemical agents/changes, pressure, etc.) into electrical/ chemical informational messengers at the info-perception interface, represented schematically by a white vertical line in the left side of Figure 1.

As the entire body is sensitive to information, the living matter can be defined as informed matter. Within the informational system of the human body (ISHB), consisting mainly in nervous network centralized into the brain - the informational processor - information is basically transmitted by electrical $\mathrm{K}^{+} / \mathrm{Na}^{+}$ions alternation near and 
along the nervous cell membrane as YES/NO (all or nothing) Bittype impulses with the same amplitude, but with different number/ frequency [8], and by chemical neurotransmitter agents within the synaptic gap of two neighbor cells. The exciting/inhibiting (YES/ NO) nature of neurotransmitters determine among other functions the mood (disposition/affective) states [9], with "Good/Bad" interpretation effect, according to personal and species experience. The interpretation of these signals is achieved at the info-interpretation interface, marked in the left side of Fig.1 as a second vertical white line. According to the brief description of IMHB presented above, the regions of the brain are specialized by functions, connected with corresponding zones of the body [10], marked by dashed horizontal lines in Figure 1, representing distinct categories of information: CASI is detected/interpreted as memory (Iknow (Ik)) - containing the life experience, CDC as decision/judgment (Iwant (Iw)), IES as feeling/ emotions (Ilove (Il) - the driving force of living, MIS as self-status/ power/vitality (Iam (Ia)), GTS as reproduction/sexual activity (Icreate (Ic)), IGG as inherited predispositions/abilities (Icreated (Icd), IC as beliefs, the most consolidated/stable form of decision/judgment criteria (Ibelieve (Ib)) [11]. IC is also involved in the mystic/religious and near/death experiences, as it was shown earlier $[12,13]$.

The primary perceived information transmitted by the informational circuits network from distinct informational sources operated by CASI/Ik is further processed by the brain. The thalamus plays an imprescriptible role of info-integration during this process, as a central hub/distributer. The information is transduced/detected at the info-detection interface on the mental "screen", which is the prefrontal cortex, where the virtual information received from the environment are perceived as forms, colors, sounds, movements, smells, touches, etc. The functioning of the cognitive centers, connected to sensors/ transducers/interpreters of information, allows the composition/ transposition of the external reality into an internal awareness/ consciousness relational state. A further processing of information allows an adaptive decision by the intervention of the $\mathrm{CDC} / \mathrm{Iw}$, as a reactive response expressed by attitude $[14,15]$, which consists in both vocal and corporal expression of information output.

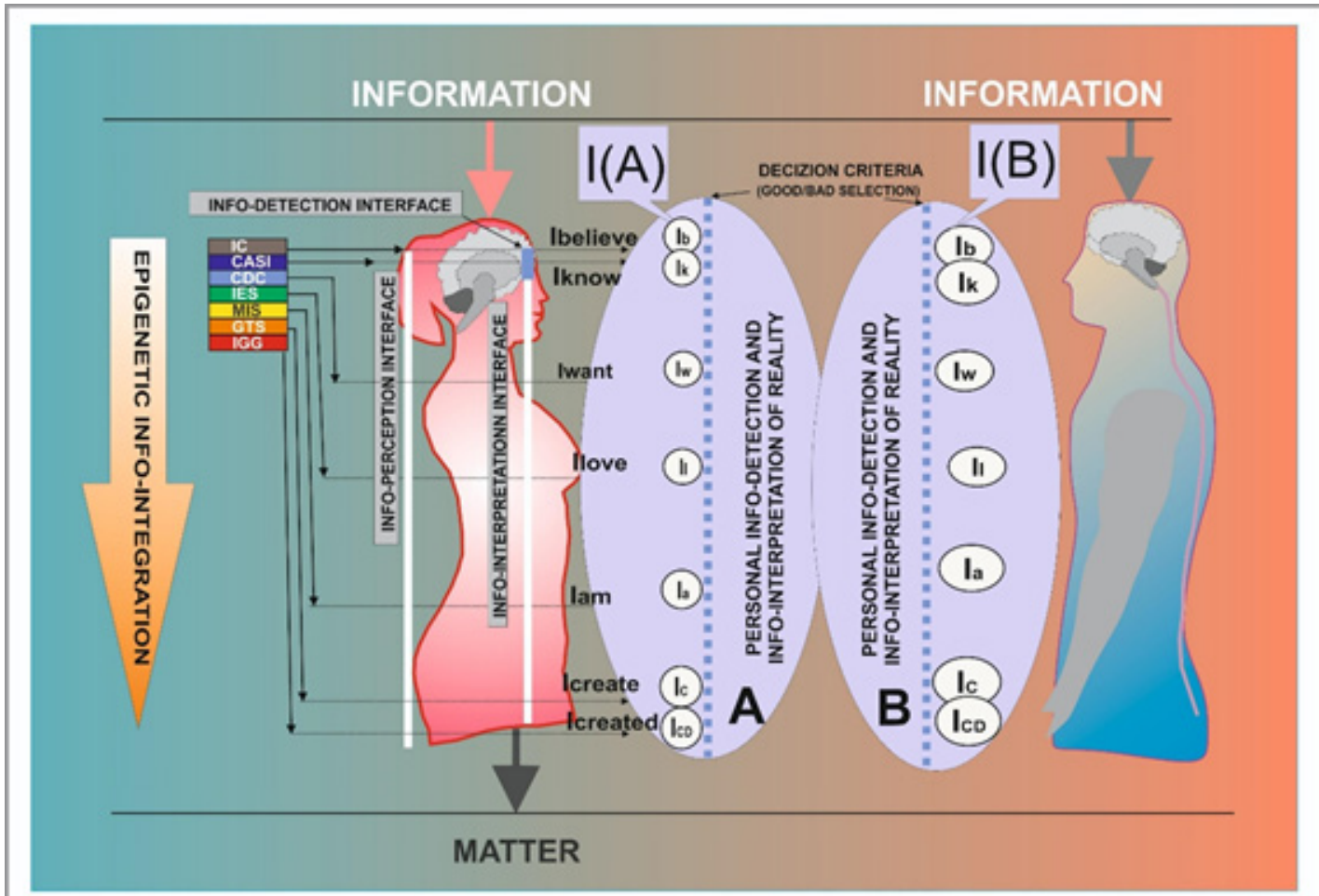

Figure 1: Schematic representation of the informational system of the human body and its corresponding components, projected in mind as cognitive centers.

The expression by language is a superior step of info-processing at human, operable at the info-interpretation interface by means of the learned specific dictionary/language. This is a virtual information, consisting in significance/meaning concepts and symbols, allowing the inter/intra-communication with collectivity and with own person. The decision operated by CDC/Iw is actually a result of the activities of all other informational cognitive centers of consciousness, all of them communicating to the central processor the momentary state, i.e. CASI/Ik on the previous life experience for analytic/comparative judgement, IC intervening with belief/trust selective criteria, IES/Il with the emotional/affective co-participation/reaction, MIS/Ia with self-status/(power) availability, GTS with social/sexual basis, IGG with the natural/inherited disposition. It is important to note here that the activity of GTS/Ic and IGG/Icd includes not only the natural/genetic activity, but also the acquired behavior by education, especially during the first years of life and adolescence/youngness period, when the nervous system and neural interconnections are still under structural (re)configuration. The GTS/Ic activity of parents is not limited therefore to the biological process. From psychological point of view, the parents' intervention within the frame of the family environment, with love/affection and careful education, is determinant for the formation of IGG/Icd of the new generation, transmitting the beliefs, culture and life orientation with features added to the native inherited info-genetic substrate of the children, useful then during the entire lifespan of the future adults. Education is a dynamic, complex process of info-transmission/communication $(=>)$ of parents experience (CASI/Ik) by CDC/Iw to their offspring.

The decision making and judgment $(\mathrm{CDC} / \mathrm{Ik})$ are informational processes achieved on the basis of comparative analysis with previous experience as/and decision criteria, allowing acceptance/rejection operations on the chain of analysis and the elaboration of a final conclusive decision. The acquirement of new information could contribute to the change of decision, and plays always a determinant role for the guiding of the next direction of behavior mode/model. The connection to reality by the cognitive centers is therefore of a major importance for the life as a dynamic system, both for the adjustment/ adaptation of the own plans/projects/behavior to the existing 
(dynamic) reality of environment, and for the internal availability and "navigation" criteria, based on inherited/acquired mentality, culture, beliefs and level of cultivation. The dynamics of life require therefore the permanent adjustment of the judgment criteria and of the judgment prototypes/stereotypes. The brain evolution itself is an indubitable example of such a necessity [16]. The basic process of the long-term adaptation is determined by epigenetic mechanisms, represented schematically by the big arrow in the left side of Figure 1 , which shows the integration of the insistent/repetitive/permanent environmental received information (on whether/food resources or other cues), from CASI to GTS, transferred finally to the IGG of the new generation [17].

The judgment is therefore an informational process based on YES/NO (Bit-type) elemental decisions, no matter their number used toward the achievement of a final firm decision. The life provides Good/ Bad experiences, which become decision criteria for further similar situations. The wisdom is an attribute of informed and experienced persons: the wisdom people are winsome people. As the life experience and inherited abilities/predispositions are different for different individuals, the decision criteria are different as well, so the perception of reality is different for each of us. The concepts like beauty/ugly for instance are relative, they depends on the own personal evaluation, i.e. the beauty depends on the "eyes which look" at it. However, for all of us, the brain circuit of beauty is distinct from the ugly circuit, the first one depends on judgment performances/habits operated in the prefrontal cortex, while the other is related to the automatic ancestral alarm/danger system - amygdala [18].

The quantity of data accumulated in memory (supported by the activity of CASI) represents the life memorized experience, as it is shown in Figure 1 right side by the areas A and B. This quantity includes the decisions/judgements operated by $\mathrm{CDC} / \mathrm{Iw}$, feelings/ emotions/emo-states operated by IES/Il, the healthy/power/selfesteem states operated by MIS/Iam, sociability/family/sexual behavior operated by GTS/Ic, inherited/epigenetic acquirements operated by IGG/Icd, info-selection criteria/beliefs operated by IC/Ib as Good/ $\mathrm{Bad}$ (YES/NO acceptance/rejection) alternatives, as it is represented in Fig. 1 for the individuals A and B. The confrontation/collaboration/ complementary activity of the structural/informational components assure the hemostatic equilibrium of the body, as a healthy axis of the dynamic info-matter system, which is the human living structure. The adaptation to the environment stimuli determines the orientation/ "navigation" on the life trajectory among the Good/Bad events/ experiences, within the limit of toleration of the organism and according to the specific personal capacity and training. Such opposite options assures the psyche equilibrium, the deviation toward any of two extreme directions leading to a need of compensation to opposite direction in order to attain the equilibrium state, but also dysfunctions of the nervous system and personal/social behavior if the deviation is experienced repetitively/intensively for a long-time period.

According to the above discussion, the informational system of the human body could be defined as: $\mathrm{ISHB}=\mathrm{CASI}+\mathrm{CDC}+\mathrm{IES}+\mathrm{MIS}+\mathrm{GTS}+\mathrm{IGG}+\mathrm{IC}$, and the corresponding detection in mind generates consciousness, as a state of awareness in relation with external and internal reality, expressed by the activity of the cognitive centers I=Ik+Iw+Il+Ia+Ic+Icd+Ib, where I is the self, different each of others (i.e. $\mathrm{I}(\mathrm{A})=/=\mathrm{I}(\mathrm{B})$ ) (Figure 1 ). The cohabitation of two or more persons or parents/sons-daughters creates a superposition of a part of the informational data, as represented schematically in Fig. 1 central zone. The total quantity $\mathrm{Q}$ of data (Information) in terms of information sets, can be expressed in this case as: $Q=Q(A) U$ Q(B) - $Q(A) \cap Q(B)$, where the symbol $U$ represents reunion of sets and $\cap$ intersection of sets. The same conditions create psychological links between partners, either being close or faraway each others, like the relation between quantic entangled particles [19].
The activity of GTS and IGG can be also described by informational processes but in a distinct "language" than that of the output information, based on the "encrypted"/codified/"embodied" information in deoxyribonucleic (DNA) and ribonucleic acid (RNA) in genes, from which various sequences of the four letter "alphabet" (the nucleotides adenine, thymine, guanine, cytosine in DNA) are communicated as various instructional "words" to mRNA (messenger RNA) to built finally the necessary proteins of the organism, assisted by amino acids and other specific forms of RNA [20]. Such type of information related with the structuration/destructuration of matter can be defined as matter-related information.

The merits of this informational model are the following: (1) the identification of the basic structural functions of the brain as informational sources of the psychological and behavioral expression, on the basis of the suitable use of the informational concepts, referring basically to virtual (conceptual) and matter-related information; (2) the unified description of various specific phenomena in the body in terms of information, independ3ntly on the information source; (3) the neuro-correlation between the informational functions and corresponding brain/body areas support; (4) the unification under the concept of information of all theories of psycological and behavior science cited above, i.e. cognition - especially operated by CASI/Ik and CDC/Iw, feelings by IES/Il, "desires" in the Freud's view by MIS/Ia (metabolic signals), GTS/Ic (sexual signals), IGG/ Icd (inherited/acquired predispositions/prototypes), humanistic concepts by the individualization of self ("I") with distinct properties in distinct individuals $(\mathrm{I}(\mathrm{A})=/=\mathrm{I}(\mathrm{B})$ ), the behavioral theory concerning the responsivity to environmental stimuli, by CASI/Ik including perception and CDC/Iw + IES/Il reactivity for short-term adaptation, and epigenetic long-term adaptation (GTS/Ic =>IGG/ Icd), biologic theory by GTS/Ic and IGG/Icd activity. Moreover, the model of the ISHB explains the operability of the brain in terms of information and the relation with the psychological and behavioral expression, allowing the successful understanding/solving of older problems like mind-body [21] and nature-nurture relation [22,23], certainty/uncertainty in cognitive-sentient exploration of reality [24], the information-matter bipolarity of the human organism [7], of the living structures $[25,26]$ and of the corresponding fundamental info-transmission circuits in neuroscience [25], the sharp definition and evaluation/quantification of attitude $[14,15]$, the info-relational cognitive operability of the anterior and posterior cingulate cortex [16,27-29] - a problem still not understood up to date in the brain sciences, the hierarchical brain organization/evolution in neurology/ neurosciences/life sciences [16], the relation with cognitive therapy in aging neurorehability [30], the mental aggressive operability [31], the pathological expression and circuits in addiction/mood disorders in psychiatry [9] and the understanding of the mind-body equilibrium and health [32]. This model opens also new ways of investigation in biotechnology/biomedicine [26,33], and through the introduction/ application of big data analysis and prediction concepts [34], in biostatistic/biometrics applications [35].

\section{Acknowledgment}

To my family, to Adrian Gaiseanu and Ana-Maria Gaiseanu.

\section{Conflict of Interest}

No financial or conflict of interests.

\section{References}

1. Seunagal Gabrielle (2021) Five Key Theories In Psychology, https:// www.betterhelp.com/advice/psychologists/five-key-theories-inpsychology/.

2. Joan d’Àvila Juanola (2020) Desire in Freud, in Desire and Human Flourishing - Perspectives from Positive Psychology. Moral Education 
and Virtue Ethic; Magdalena Bosch: Springer; pp. 291-306.

3. Gaiseanu Florin (2021) Information as an essential component of the biological structures and their informational organization. Journal of Microbiology \& Biotechnology 6(2): 1-9.

4. Gaiseanu Florin (2021) Advanced Results in Biological Researches: Informational Structure and Info-Operability of the Living Systems. Journal of Biotechnology \& Bioresearch 3(3): 1-4.

5. Gaiseanu Florin (2020) Informational structure of the living systems: From philosophy to informational modeling. Philosophy Study 10(12): 795-806.

6. Gaiseanu Florin (2021) Information in the Universal Triangle of Reality for Non-living/Living Structures: From Philosophy to Neuro/Life Sciences. Philosophy Study 11(8): 607-621.

7. Gaiseanu Florin (2020) Information-Matter Bipolarity of the Human Organism and Its Fundamental Circuits: From Philosophy to Physics/ Neurosciences-Based Modeling. Philosophy Study 10(2): 107-118.

8. Gaiseanu Florin (2021) Information, Info-Creational Field, Creativity and Creation, According to the Informational Model of Consciousness. International Journal on Neuropsychology and Behavioural Sciences 2(3): $75-80$.

9. Gaiseanu Florin (2021) Pathological expression and circuits in addiction and mood disorders: Informational relation with the brain and infotherapy. EC Neurology 13(8): 1-12.

10. Gaiseanu Florin (2019) Informational Neuro-Connections of the Brain with the Body Supporting the Informational Model of Consciousness, Archives in Neurology \& Neuroscience 4(1): 1-6.

11. Gaiseanu Florin (2018) Destiny or Free Will Decision? A Life Overview From the Perspective of an Informational Modeling of Consciousness Part II: Attitude and Decision Criteria, Free Will and Destiny. Gerontology \& Geriatric Studies 4(1): 1-7.

12. Gaiseanu Florin (2017) Quantum-Assisted Process of Disembody Under Near-Death Conditions: An Informational-Field Support Model. NeuroQuantology 15(1): 4-9.

13. Gaiseanu Florin (2017) An Information Based Model of Consciousness Fully Explaining the Mind Normal/Paranormal Properties. NeuroQuantology 15(2): 132-140.

14. Gaiseanu Florin (2020) Attitude as an Expressible Info-Operational Reaction to a Perceived/Purposed Object/Objective. International Journal on Neuropsychology and Behavioural Sciences 1(1): 12-16.

15. Gaiseanu Florin (2021) Evaluating attitude and behavior: An infooperational procedure related/supported by the cognitive centers of mind. International Journal on Neuropsychology and Behavioural Sciences 2(1): 1-5.

16. Gaiseanu Florin (2020) Information Based Hierarchical Brain Organization/Evolution from the Perspective of the Informational Model of Consciousness. Archives in Neurology \& Neuroscience 7(5): $1-6$.

17. Gaiseanu Florin (2019) Epigenetic Information-Body Interaction and Information-Assisted Evolution from the Perspective of the Informational Model of Consciousness. Archives in Biomedical Engineering \& Biotechnology, 2(2): 1-6.

18. Gaiseanu Florin (2021) Neuropsychological response to information of beauty/ugly brain circuits according to the informational model of consciousness. International Journal on Neuropsychology and Behavioural Sciences 2(2): 55-59.

19. Gaiseanu Florin (2020) Physics of Consciousness and Life: The Informational Model of Consciousness. Information in Neurosciences, Biocom- puters and Biosystems.

20. Gaiseanu Florin (2020) What is Life: An Informational Model of the Living Structures. Biochemistry and Molecular Biology 5(2): 18-28.

21. Gaiseanu Florin (2021) Solution to the Mind-Body Relation Problem: Information. Philosophy Study 11(1): 42-55.

22. Gaiseanu Florin (2019) The Silent Voice of Those Who are no Longer: Transgenerational Transmission of Information from the Perspective of the Informational Model of Consciousness. Gerontology \& Geriatric Studies 5(1): 482-488.

23. Gaiseanu Florin (2019) Language Patterns and Cognitive-Sentient Reality: Certainty/Uncertainty in Cognitive-Sentient Exploration of Reality. Stephen Brock Schafer (Eds). Media Models to Foster Collective Human Coherence in the PSYCHecology; USA.

24. Gaiseanu Florin (2021) New Perspectives in Biomedical Engineering and Biotechnology: Information in Human and Biological Structures. Archives in Biomedical Engineering and Biotechnology 6(1): 1-3.

25. Gaiseanu Florin (2021) Evolution and Development of the Information Concept in Biological Systems: From Empirical Description to Informational Modeling of the Living Structures. Philosophy Study 11(7): 501-516.

26. Gaiseanu Florin (2021) New Perspectives in Biomedical Engineering and Biotechnology: Information in Human and Biological Structures, Archives in Biomedical Engineering and Biotechnology, 6(1): 1-3. ABEB.MS.ID.000633. DOI: 10.33552/ABEB.2021.06.000633.

27. Gaiseanu Florin (2021) Informational Operability of the Brain: Novel Approaching Perspectives in Neuroscience and Neurology(Guest Editorial), EC Neurology 13 (12): 50-54. https://www.ecronicon.com/ ecne/pdf/ECNE-13-00983.pdf

28. Gaiseanu Florin (2019) The Informational Model of Consciousness: Mechanisms of Embodiment/Disembodiment of Information, NeuroQuantology, 17(4), 1-17.

29. Gaiseanu Florin (2020) Info-Relational Cognitive Operability of the Posterior Cingulate Cortex According to the Informational Model of Consciousness, International Journal of Psychological and Brain Sciences, 5(4): 61-68.

30. Gaiseanu Florin (2021) Multitask Music-Based Therapy Optimization in Aging Neurorehability by Activation of the Informational Cognitive Centers of Consciousness, Gerontology\&Geriatrics Studies, 6(3), 621625.

31. Gaiseanu Florin (2021). Mental aggressive operability from informational perspective: A deterrence manifesto, EC Neurology, 13(4), 31-39.

32. Gaiseanu Florin (2020) Informationally-Assisted Equilibrium and Health: Specific ACC Contribution from the Perspective of the Informational Model of Consciousness, EC Psychology and Psychiatry J., 9(5), 37-49.

33. Gaiseanu Florin (2021) Human as an Informational Device, Archives in Biomedical Engineering \& Biotechnology 6(1): 1-8. ABEB. MS.ID.000629. DOI: 10.33552/ABEB.2021.06.000629.

34. Filip Florin Gheorghe (2020) DSS-A class of evolving information systems. In: G Dzemyda, J Bernatavičienė, J Kacprzyk (Eds.), Data science: New issue, challenges and applications. Springer, pp. 253-277.

35. Gaiseanu Florin (2021) Information in Biological Structures and Big Data Assisted Prediction as Informational Biostatistics/Biometric Tool for Pandemic COVID-19 Investigation, Annals of Biostatistics \& Biometric Applications, 4(3): 1-3. ABBA.MS.ID.000592. DOI: 10.33552/ ABBA.2020.04.000592. 